\title{
Dietary triggers of gut inflammation following exclusive enteral nutrition in children with Crohn's disease: a pilot study
}

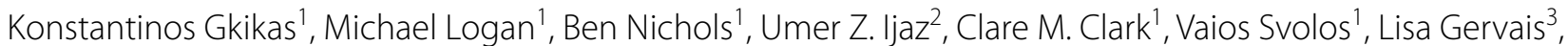
Hazel Duncan ${ }^{4}$, Vikki Garrick ${ }^{3}$, Lee Curtis ${ }^{3}$, Elaine Buchanan ${ }^{3}$, Tracey Cardigan ${ }^{3}$, Lawrence Armstrong ${ }^{4}$, Caroline Delahunty ${ }^{5}$, Diana M. Flynn ${ }^{3}$, Andrew R. Barclay ${ }^{3}$, Rachel Tayler ${ }^{3}$, Simon Milling ${ }^{6}$, Richard Hansen ${ }^{3}$, Richard K. Russell ${ }^{7}$ and Konstantinos Gerasimidis ${ }^{{ }^{*}}$

\begin{abstract}
Background: The anti-inflammatory effect of exclusive enteral nutrition on the gut of children with Crohn's disease is rapidly lost after food reintroduction. This study assessed disease dietary triggers following successful treatment with exclusive enteral nutrition.

Methods: Nutrient intake, dietary patterns and dietary biomarkers in faeces (gluten immunogenic peptides, undigestible starch, short chain fatty acids) were assessed in 14 children with Crohn's disease during early food reintroduction, following exclusive enteral nutrition. Groups above (Group A) and below (Group B) the median levels of faecal calprotectin after food reintroduction were assigned for comparative analysis.

Results: Intakes of fibre, gluten-containing cereals and red and processed meat were significantly higher in Group A than Group B; (median [Q1, Q3], g/day; Fibre: 12.1 [11.2, 19.9] vs. 9.9 [7.6, 12.1], $p=0.03$; Red and processed meat: $151[66.7,190]$ vs. $63.3[21.7,67], p=0.02$; gluten-containing cereals: $289[207,402]$ vs. 203 [61, 232], p=0.035). A diet consisting of cereals and meat products was predictive ( $92 \%$ accuracy) of higher faecal calprotectin levels after food reintroduction. In faeces, butyrate levels, expressed as absolute concentration and relative abundance, were higher in Group A than Group B by $28.4 \mu \mathrm{mol} / \mathrm{g}(p=0.015)$ and $6.4 \%(p=0.008)$, respectively. Levels of gluten immunogenic peptide and starch in faeces did not differ between the two groups.
\end{abstract}

Conclusions: This pilot study identified potential dietary triggers of gut inflammation in children with Crohn's disease after food reintroduction following treatment with exclusive enteral nutrition.

Trial registration: Clinical trials.gov registration number: NCT02341248; Clinical trials.gov URL: https://clinicaltrials.gov/ ct2/show/NCT02341248 (retrospectively registered).

Keywords: Crohn's disease, Food reintroduction, Dietary triggers, Faecal calprotectin, Gluten, Fibre, Meat, Short chain fatty acids

*Correspondence: konstantinos.gerasimidis@glasgow.ac.uk

${ }^{1}$ Human Nutrition, School of Medicine, Dentistry and Nursing, College of Medical, Veterinary and Life Sciences, University of Glasgow, Glasgow, UK

Full list of author information is available at the end of the article

\section{Introduction}

The increasing incidence of Crohn's disease (CD) in societies in economic transition, suggests that environmental factors, including a Western diet are major contributors to the disease pathogenesis [1]. In nutritional epidemiology, adherence to the principles of the Mediterranean 
diet has protected against development of $\mathrm{CD}[2,3]$ whereas food additives have been implicated in the development of intestinal inflammation in animal models [4].

Exclusive enteral nutrition (EEN) is the only established dietary treatment for active $\mathrm{CD}$ in children $[5,6]$. In addition to symptom improvement, EEN reduces faecal calprotectin (FC) levels by a mean of $50 \%$ by the end of treatment [7]. However, we have recently observed a rapid increase in FC within the first 17 days of food reintroduction, following treatment with EEN; an effect which preceded any noticeable changes in clinical disease activity [8]. This intriguing observation suggests that reintroduction of certain dietary components, after return to habitual diet, provokes recurrence of intestinal inflammation.

In the current study, we performed detailed assessment of the diet of children with CD during this early phase of food reintroduction, following successful EEN, and explored relationships with FC. We concentrated our efforts on this critical food reintroduction period, which provided us with a unique timeframe to explore dietary disease triggers in a population with homogeneous characteristics of disease activity, type, and duration of preceding EEN treatment at study enrolment.

\section{Methods}

\section{Patients}

Children (aged 3-17 years) with a new diagnosis of CD or with disease in relapse, who initiated an 8-week course of EEN were recruited from the Royal Hospital for Children, Glasgow and neighbouring hospitals, as described previously [9]. A faecal sample per patient was collected during early food reintroduction, between 15 and 30 days after EEN completion or at the earliest most convenient time for the patients.

Disease activity was assessed with the weighted Paediatric Crohn's Disease Activity Index (wPCDAI) [10]. In the current study, we included a subset of patients recruited in the study by Logan et al. [9], who achieved both clinical remission at EEN completion (wPC$\mathrm{DAI}<12.5)$ and experienced a clinically significant drop in FC, defined by a decrease of $\geq 50 \%$ or $\geq 500 \mathrm{mg} / \mathrm{kg}$ from levels at EEN initiation (Additional file 1: Fig. S1). Patients who did not enter clinical remission or did not provide a faecal sample or dietary records were excluded. Information about disease phenotype (Paris classification) [11] and concomitant treatment was collected from medical notes.

\section{Dietary assessment}

Participants recorded their diet for three days prior to faecal sample collection, including intake of enteral nutrition as maintenance treatment (MEN), with 3-day food diaries, estimating weight of consumed foods using household measures. MEN was prescribed to provide $20-25 \%$ of daily energy requirements. No specific food reintroduction guidance was provided to patients after EEN cessation. Dietary records were analysed using the WinDiets software (WinDiets version 10, Robert Gordon University, Aberdeen, UK) for calculation of energy and nutrient intake.

Dietary patterns were assessed by classification of individual foods in groups based on the grouping applied in the UK National Dietary and Nutrition Survey (NDNS) [12]. Individual foods were assigned to the subsidiary NDNS food groups (level 3, e.g., rice) and the subsidiary groups were combined to form the main NDNS groups (level 2, e.g., pasta, rice, and other cereals), which then formed the larger level food groups (e.g., cereal and cereal products) (level 1) (Additional file 2: Fig. S2). Cereal products were divided to gluten and non-gluten containing cereal products. Meat subtypes were also grouped under the categories: 'red meat' and 'processed meat' separately, and also in a combined 'red and processed meat' category based on the World Cancer Research Fund definitions [13], since the consumption of both types of meat has been implicated in the pathogenesis of Inflammatory bowel diseases (IBD) [14, 15]. Nutrient intake was expressed in absolute mass (grams). Energy intake was further expressed as percentage of estimated average requirements (EAR), macronutrients as percentages of total energy intake, and protein and micronutrients as percentages of reference nutrient intakes (RNI) [16]. Dietary fibre was defined as non-starch polysaccharides, in accordance with the Englyst method.

\section{Faecal sample collection and faecal calprotectin}

The entire bowel movement was collected within $4 \mathrm{~h}$ of defecation and was transferred to the laboratory on ice under anaerobic conditions (Oxoid AnaeroGen Sachet; ThermoFisher Scientific)[9]. Samples were homogenised and aliquots were stored in $-80{ }^{\circ} \mathrm{C}$. Faecal calprotectin was measured using the CALP0170 kit (CalproLab, Lysaker, Norway) [17].

\section{Faecal biomarkers of food intake}

Short and branched chain fatty acids (SCFA, BCFA) were measured using gas chromatography (Agilent 7890A) [18], and were used as proxy biomarkers of fermentable dietary fibre and protein intake, respectively. Starch (Megazyme, Ireland) and gluten immunogenic peptides (GIP, Biomedal, S.L., Spain) were measured in faeces, as biomarkers of non-fermented resistant starch and gluten intake, respectively [19], according to the manufacturers' instructions. For the measurement of faecal starch, 
samples were initially lyophilised and homogenised thoroughly using a pestle and mortar.

\section{Statistical considerations}

Statistical analysis was performed using Minitab, Version 18 (Minitab Ltd, Coventry, UK) and R (version 3.5.3). Patients were divided into two equally numbered groups based on their FC levels during food reintroduction. Patients with an FC above the median concentration after food reintroduction were assigned to Group A (Above) and patients with an FC below the median concentration to Group B (Below). Continuous data were expressed as medians with interquartile ranges $(\mathrm{Q} 1, \mathrm{Q} 3)$ and categorical data as counts with frequencies (n [\%]), unless otherwise stated. Comparisons of continuous variables between different groups were performed on Box-Cox transformed data using general linear models and Fisher's least significance difference post-hoc test. Comparisons of dietary intake and biomarker levels between the two groups were adjusted for FC levels at EEN completion (p-adj). Relationships between dietary components and FC levels were explored with Pearson and Spearman rank correlations.

For dietary pattern analysis, subset regression analysis was performed by testing all possible food group combinations. The best predictive model was selected according to recommendations given in Kassambara, 2018 [20], and was used to predict the FC group of each patient based on dietary patterns consisting of multiple food groups. In-house $\mathrm{R}$ scripts incorporating the leaps [21] and caret packages [22], were used. Specifically, we used the relative contribution of each individual food group intake to the cumulative food group intake. Food groups consumed by fewer than two patients were excluded from the analysis. After the various models were created, random forest analysis, using the randomForest package [23] was employed to select the best model based on the lowest assignment error (out-of-bag error). The relative importance of each food group intake to the predictive ability of each model was assessed by calculating the mean reduction in Gini impurity index on inclusion.

\section{Results}

\section{Anthropometric and clinical disease characteristics}

Fourteen out of 23 (61\%) paediatric patients, who had maintained symptom-free clinical remission at the time of the sample collection, provided a faecal sample and completed dietary records within (median [Q1, Q3]): 21 [15, 51] days of food reintroduction. None had commenced or were receiving another induction therapy. The median [Q1, Q3] FC for all 14 patients after food reintroduction was $900 \mathrm{mg} / \mathrm{kg}[341,1243]$. In Group A, median [Q1, Q3] FC levels were 1,181
[1024, 1781] mg/kg and in Group B $411[130,651] \mathrm{mg} /$ $\mathrm{kg}$ (Additional file 3: Fig. S3). No significant differences were observed in demographics, anthropometry, or disease characteristics between the two groups (Additional file 4: Table S1). There were no significant differences in immunomodulator use, energy intake from MEN, and the time elapsed between the end of EEN and sample collection after food reintroduction. Likewise, no significant correlation was observed between FC levels and the time elapsed between the end of EEN and sample collection after food reintroduction $(p=0.899)$.

\section{Nutrient intake}

Group A reported a significantly higher intake of fibre compared to Group B, expressed both as an absolute amount (median [Q1, Q3], grams; Group A: 12.1 $[11.2,19.9]$ vs. Group B: 9.9 [7.6, 12.1], $p=0.030, p$ adj $=0.068$ ) and as percentage of RNI (median [Q1, Q3], \%RNI; Group A: 55 [46, 99.5] versus Group B: 44.8 $[30.3,48.5], p=0.023, p$-adj $=0.071)$ (Table 1). Intakes of protein and phosphorus, expressed as \%RNI, were also significantly higher in Group A than Group B (median [Q1, Q3], \%RNI; Protein; Group A: 262 [195, 291] versus Group B: 211 [88.4, 215], $p=0.026, p$ adj $=0.069$; Phosphorus; Group A: $241[171,290]$ versus Group B: 177 [130, 191], $p=0.040, p$-adj=0.174) (Table 1).

\section{Food group intake}

Although the intake of red meat and processed meat, when assessed separately, did not differ between the two groups ( $p=0.225, p=0.125$ respectively), their cumulative intake in Group A was significantly higher compared to Group B by a median difference of $87.7 \mathrm{~g} /$ day (median [Q1, Q3], grams; Group A: 151 [66.7, 190] vs. Group B: 63.3 [21.7, 67], $p=0.030, p$-adj=0.065) (Fig. 1). Consumption of cereals and cereal products was higher in Group A, although the difference did not reach statistical significance $(p=0.08, p$-adj $=0.067)$. Nonetheless, the intake of gluten containing cereal products was higher in Group A than Group B, and in reverse, the consumption of non-gluten containing cereal products was higher in Group B (median [Q1, Q3], grams; gluten containing cereal products, Group A: 289 [207, 402] vs. Group B: 203 [61, 231], $p=0.035, p$-adj $=0.042$; non-gluten containing cereal products: Group A: $0[0,20]$ vs. Group B: 43 [33, 77], $p=0.031, p$-adj=0.083). The food groups 'alcoholic beverages', 'commercial toddlers' foods and drinks' and 'nuts and seeds' were not consumed by any of the patients and were therefore excluded from analysis. 
Table 1 Comparison of nutrient intake between patients in the two groups

\begin{tabular}{|c|c|c|c|c|}
\hline & Group A $(n=7)$ & Group B $(n=7)$ & $p$ value & $p$ value adj \\
\hline Total energy (kcal) & $2057(1916,2305)$ & $1906(1473,2252)$ & 0.365 & 0.217 \\
\hline Total energy (\% EAR) & $97.2(89.2,118)$ & $85.7(69.3,105)$ & 0.08 & 0.177 \\
\hline Fat (g) & $71.6(65.7,82.5)$ & $75.3(63,83.6)$ & 0.764 & 0.719 \\
\hline Fat (\% kcal) & $32.3(28.4,35.5)$ & $33.8(30.6,35.6)$ & 0.418 & 0.317 \\
\hline SFA (g) & $25.0(24.3,33.1)$ & $29.0(24.8,37.4)$ & 0.602 & 0.533 \\
\hline SFA (\% kcal) & $11.7(10.5,13.9)$ & $13.1(10.9,15.2)$ & 0.381 & 0.269 \\
\hline MUFA (g) & $27.1(20.9,27.7)$ & $21.8(17.5,24.5)$ & 0.237 & 0.315 \\
\hline MUFA (\% kcal) & $10.9(9.8,12.0)$ & $10.5(9.7,11.5)$ & 0.463 & 0.78 \\
\hline PUFA (g) & $11.5(9.8,12.9)$ & $9.4(6.9,10.8)$ & 0.082 & 0.236 \\
\hline PUFA (\% kcal) & $4.8(3.8,6.5)$ & $4.3(4.1,5.1)$ & 0.336 & 0.887 \\
\hline Carbohydrates (g) & $267(239,303)$ & $246(225,284)$ & 0.415 & 0.19 \\
\hline Carbohydrates (\% kcal) & $52.5(48.5,56.0)$ & $51.6(49,56.9)$ & 0.831 & 0.807 \\
\hline Sugars (g) & $97.4(84.1,105)$ & $93.3(89.1,118)$ & 0.636 & 0.393 \\
\hline Sugars (\% kcal) & $18.9(16.2,22)$ & $21.4(20.9,24.2)$ & 0.175 & 0.393 \\
\hline Dietary fibre (g) & $12.1(11.2,19.9)$ & $9.9(7.6,12.1)$ & 0.03 & 0.068 \\
\hline Dietary fibre (\% RNI) & $55(46,99.5)$ & $44.8(30.3,48.5)$ & 0.023 & 0.071 \\
\hline Starch (g) & $146(105,154)$ & $126(120,144)$ & 0.366 & 0.216 \\
\hline Protein (g) & $82.2(73.7,90.2)$ & $65.4(37.2,89.5)$ & 0.179 & 0.159 \\
\hline Protein (\% kcal) & $16.8(13.8,17.3)$ & $14.9(10.7,15.9)$ & 0.135 & 0.196 \\
\hline Protein (\% RNI) & $262(195,291)$ & $211(88.4,215)$ & 0.026 & 0.069 \\
\hline Vitamin A ( $\mu \mathrm{g})$ & $485(451,762)$ & $695(485,1000)$ & 0.709 & 0.464 \\
\hline Vitamin A (\% RNI) & $80.8(75.2,153)$ & $139(80.8,167)$ & 0.948 & 0.617 \\
\hline Vitamin D $(\mu \mathrm{g})$ & $1.2(0.9,1.6)$ & $0.9(0.6,1.2)$ & 0.536 & 0.844 \\
\hline Vitamin C (mg) & $115(62.1,127)$ & $102(77.6,117)$ & 0.909 & 0.871 \\
\hline Vitamin C (\% RNI) & $362(207,401)$ & $291(194,389)$ & 0.821 & 0.743 \\
\hline Thiamine (mg) & $1.8(1.2,2.2)$ & $1.4(0.8,1.9)$ & 0.297 & 0.483 \\
\hline Thiamine (\% RNI) & $251(171,310)$ & $159(101,212)$ & 0.068 & 0.214 \\
\hline Vitamin B2 (mg) & $1.3(1.1,1.7)$ & $1.4(1,1.8)$ & 0.608 & 0.352 \\
\hline Vitamin B2 (\% RNI) & $141(112,174)$ & $129(103,153)$ & 0.343 & 0.404 \\
\hline Vitamin B6 (mg) & $2.3(1.6,2.8)$ & $2.2(1.7,3.2)$ & 0.938 & 0.548 \\
\hline Vitamin B6 (\% RNI) & $234(141,276)$ & $184(145,267)$ & 0.63 & 0.772 \\
\hline Niacin (mg) & $34.3(26.8,42.5)$ & $32.3(25.8,37.3)$ & 0.338 & 0.323 \\
\hline Niacin (\% RNI) & $283(224,354)$ & $231(172,249)$ & 0.104 & 0.143 \\
\hline Folic acid $(\mu \mathrm{g})$ & $215(163,306)$ & $211(172,294)$ & 0.784 & 0.71 \\
\hline Folic acid (\% RNI) & $139(81.5,204)$ & $106(86,147)$ & 0.374 & 0.859 \\
\hline Vitamin B12 ( $\mu \mathrm{g})$ & $3.4(2.9,4.9)$ & $3.6(2.8,4.9)$ & 0.89 & 0.472 \\
\hline Vitamin B12 (\% RNI) & $337(195,412)$ & $324(187,414)$ & 0.828 & 0.567 \\
\hline Pantothenic acid (mg) & $4.8(4.2,5.7)$ & $4.2(3.2,6.6)$ & 0.454 & 0.708 \\
\hline Biotin $(\mu \mathrm{g})$ & $24(22.1,27)$ & $22.1(16.2,39)$ & 0.893 & 0.357 \\
\hline Vitamin E (mg) & $6.4(3.6,7.6)$ & $6.1(4.5,6.5)$ & 0.816 & 0.717 \\
\hline Sodium (mg) & $2084(1977,2834)$ & $2073(1399,2682)$ & 0.528 & 0.365 \\
\hline Sodium (\% RNI) & $130(124,177)$ & $130(87.4,168)$ & 0.528 & 0.365 \\
\hline Chloride (mg) & $3307(2368,4315)$ & $3647(2180,3756)$ & 0.484 & 0.49 \\
\hline Chloride (\% RNI) & $167(94.7,238)$ & $146(121,150)$ & 0.244 & 0.362 \\
\hline Potassium (mg) & $2293(1930,3068)$ & $2326(1807,2617)$ & 0.468 & 0.651 \\
\hline Potassium (\% RNI) & $115(99,223)$ & $84.4(72.4,96)$ & 0.127 & 0.4 \\
\hline Calcium (mg) & $1068(718,1323)$ & $1103(645,1470)$ & 0.942 & 0.951 \\
\hline Calcium (\% RNI) & $128(93.3,157.2)$ & $102(79.6,115)$ & 0.142 & 0.571 \\
\hline Phosphorus (mg) & $1241(1005,1323)$ & $1103(814,1470)$ & 0.527 & 0.821 \\
\hline
\end{tabular}


Table 1 (continued)

\begin{tabular}{|c|c|c|c|c|}
\hline & Group A $(n=7)$ & Group B $(n=7)$ & $p$ value & $p$ value adj \\
\hline Phosphorus (\% RNI) & $241(171,290)$ & $177(130,191)$ & 0.04 & 0.174 \\
\hline Magnesium (mg) & $262(187,285)$ & $207(179,270)$ & 0.53 & 0.981 \\
\hline Magnesium (\% RNI) & $101(66.8,142)$ & $82.3(68.8,90.1)$ & 0.218 & 0.692 \\
\hline Iron (mg) & $10.9(7.9,14.1)$ & $8.9(8.2,12.7)$ & 0.414 & 0.979 \\
\hline Iron (\% RNI) & $114(69.6,148)$ & $76.4(73.5,112)$ & 0.257 & 0.886 \\
\hline Zinc (mg) & $11.4(6.70,13.8)$ & $7.8(6.35,10.2)$ & 0.393 & 0.745 \\
\hline Zinc (\% RNI) & $153(95.7,164)$ & $106(86.7,117)$ & 0.179 & 0.474 \\
\hline Copper (mg) & $0.99(0.95,1.55)$ & $1.07(0.76,1.26)$ & 0.443 & 0.857 \\
\hline Copper (\% RNI) & $136(95,221)$ & $111(95.4,134)$ & 0.268 & 0.964 \\
\hline lodine $(\mu \mathrm{g})$ & $91.7(80,117)$ & $117(90.3,162)$ & 0.468 & 0.322 \\
\hline lodine (\% RNI) & $77.4(70.5,106)$ & $102(64.5,124)$ & 0.811 & 0.46 \\
\hline Selenium ( $\mu \mathrm{g})$ & $42(31,49.3)$ & $30(24,54.7)$ & 0.382 & 0.762 \\
\hline Selenium (\% RNI) & $110(67.4,153)$ & $73.3(44.4,121)$ & 0.159 & 0.531 \\
\hline Cholesterol (mg) & $208(160,286)$ & $203(73,268)$ & 0.464 & 0.506 \\
\hline Carotene (ug) & $1561(325,3497)$ & $950(179,1939)$ & 0.370 & 0.415 \\
\hline
\end{tabular}

Group A: patients with faecal calprotectin levels above the median levels at food reintroduction, Group B: patients with faecal calprotectin values below the median levels at food reintroduction. $p$ value adj: $p$ value adjusted for faecal calprotectin levels at the end of EEN. Values are presented as medians (Q1, Q3). EAR: Estimated average requirement, MUFA: Monounsaturated fatty acids, PUFA: Polyunsaturated fatty acids, RNI: Reference nutrient intake, SFA: Saturated fatty acids

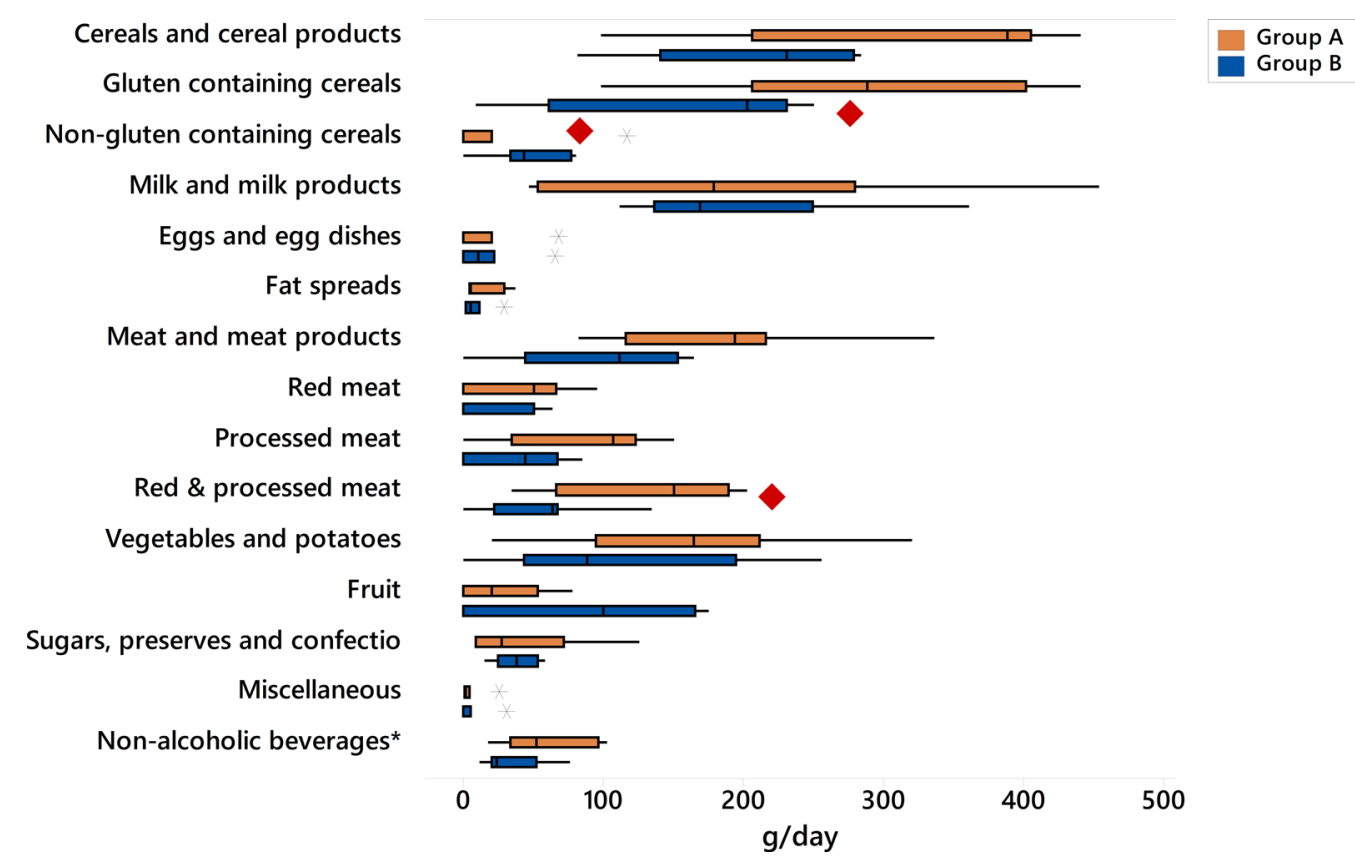

Fig. 1 Comparison of food group intake between the two groups in children with Crohn's Disease during food reintroduction. Group A: patients with faecal calprotectin levels above the median levels at food reintroduction, Group B: patients with faecal calprotectin values below the median levels at food reintroduction * Intake of non-alcoholic beverages has been divided by 10 for both groups for better visualisation. Red diamond indicates significant differences between Group A and B $(p=0.03)$

\section{Relationships between nutrients, food groups, dietary patterns, and levels of FC}

To explore linear relationships, as a proxy of doseresponse associations between dietary intake parameters and FC levels, we performed correlation analysis
(Fig. 2, Additional file 4: Tables S2 and S3). Protein intake (\% RNI) $(\mathrm{r}=0.54, p=0.047)$, thiamine $(\mathrm{mg})$ (Pearson $\mathrm{r}=0.57, p=0.033)$ and niacin (\%RNI $(\mathrm{r}=0.61, \mathrm{p}=0.02)$ had a moderate-strong positive correlation with FC (Fig. 2). 

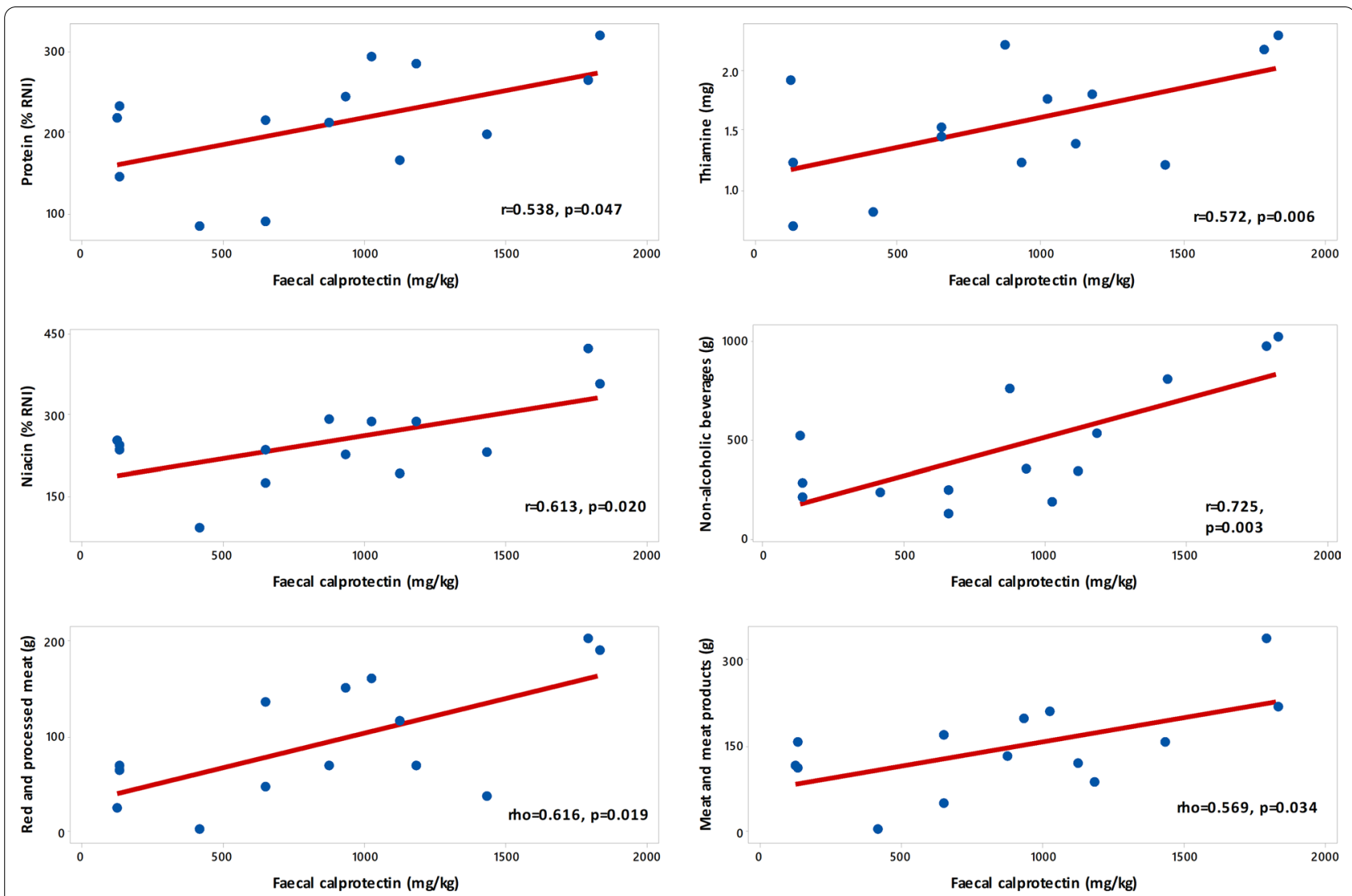

Fig. 2 Correlations between nutrient and food intake with faecal calprotectin levels in children with Crohn's Disease after food reintroduction

Moderate-strong significant positive correlations with FC levels were also observed between the intakes of red and processed meat (Spearman's rho $=0.62, p=0.019$ ), meat and meat products (Spearman's rho $=0.57$, $p=0.034)$ and the intake of non-alcoholic beverages which included juices, and fizzy drinks (Pearson $r=0.73$, $p=0.003$ ) (Fig. 2).

The contribution of dietary patterns in the prediction of FC levels was assessed using food-group models (Fig. 3). Cereals and meat products showed a positive association with the assignment of patients in Group A, whilst for eggs, fruits and fruit products, the association was negative. Random forest analysis showed that the model with the lowest 'out-of-bag' error included cereal, meat and their products, and could predict the correct classification of patients to their respective FC group with a high accuracy of over 92\% (error rate: 7.7\%, Model 2, Fig. 3). The contribution of meat and meat products to the predictive ability of Model 2 was $62 \%$, and that of cereals $38 \%$, suggesting that a dietary pattern high in cereals and meat products was associated with higher levels of FC after food reintroduction. The model with the second lowest error rate included in addition fruits and eggs, which showed a negative (i.e., a protective) association with faecal calprotectin levels (error rate: 15.4\%, Model 3, Fig. 3).

\section{Diet-related biomarkers in faeces}

Butyrate levels, expressed either as absolute concentration $(\mu \mathrm{mol} / \mathrm{g})$ or as relative proportion (\%), were significantly higher in patients with higher FC levels (Group A), compared to patients with lower FC levels (Group B) by a median difference of $28.4 \mu \mathrm{mol} / \mathrm{g}$ and $6.4 \%$ respectively (median [Q1, Q3], $\mu \mathrm{mol} / \mathrm{g}:$ Group A: 62.5 [41.8, 114] vs. Group B: 34.1 [29.2, 49.6], $p=0.016, p$-adj=0.015; \%; Group A: 15.1 [11.2, 17.8] vs. Group B: 8.7 [8.6, 9.12], $p=0.008, p$-adj $=0.016$ ), Table 2 . The concentration of acetate, propionate and of BCFA did not differ between the two groups. Faecal GIP levels did not significantly differ between the two groups (median [Q1, Q3], ng/ $\mu$ g; Group A: 1,250 [1,250, 1,250] vs. Group B: 1,250 [78, $1,250], p=0.121, p$-adj=0.211), although most patients in Group A had GIP measurements above the upper detection limit of the assay, suggesting high gluten intake. 


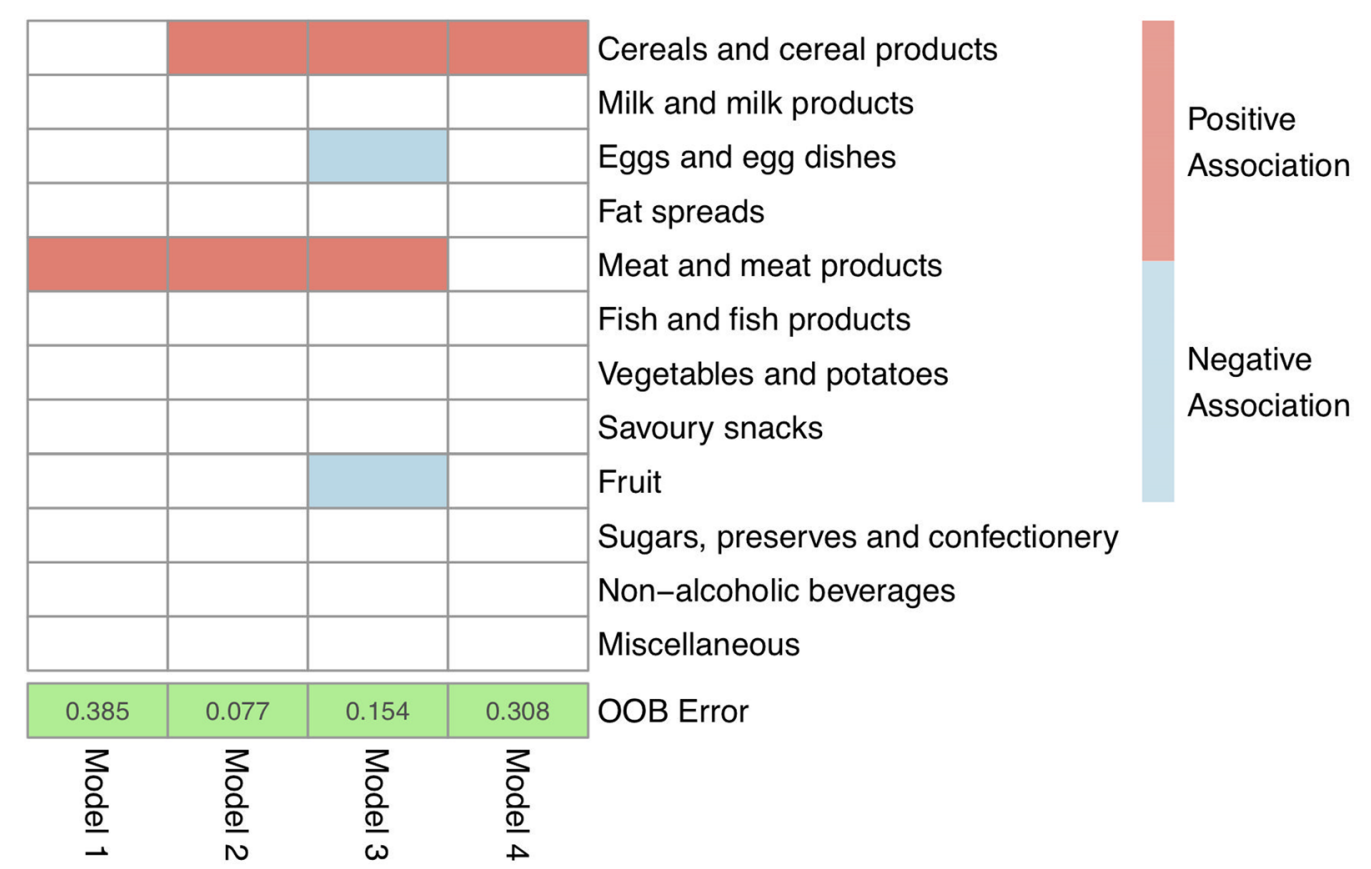

Fig. 3 Results from subset regression analysis showing food-group models predicting classification of patients in the two FC groups. Food groups coloured with red indicate positive association (i.e., harmful) and with blue negative (i.e., beneficial) association with assignment of patients in the group with higher FC levels during food reintroduction (Group A) (higher FC levels during food reintroduction). OOB error: Out of bag error rate, showing percentage of misclassification of patients to their respective FC groups (Group A/B) for each different food-group model identified from random forest analysis. Fish and fish-related products were removed from analysis, as they were consumed by one patient

Table 2 Comparison of concentration of SCFA in faeces between patients in the two groups

\begin{tabular}{|c|c|c|c|c|}
\hline SCFA & Group A $(n=6)$ & Group B $(n=7)$ & $p$ value & $p$ value adj \\
\hline Faecal water content (\%) & $74(70,80.5)$ & $73.2(65.7,80)$ & 0.601 & 0.340 \\
\hline Acetate $(\mu \mathrm{mol} / \mathrm{g})$ & $255(238,341)$ & $304(223,385)$ & 0.716 & 0.959 \\
\hline Propionate ( $\mu \mathrm{mol} / \mathrm{g}$ ) & $80.5(70.5,143)$ & $72.1(46.3,109)$ & 0.367 & 0.126 \\
\hline Isobutyrate ( $\mu \mathrm{mol} / \mathrm{g})$ & $10.9(7.6,16.7)$ & $8.70(6.1,9.9)$ & 0.121 & 0.174 \\
\hline Butyrate $(\mu \mathrm{mol} / \mathrm{g})$ & $62.5(41.8,114)$ & $34.1(29.2,49.6)$ & 0.016 & 0.015 \\
\hline Isovalerate $(\mu \mathrm{mol} / \mathrm{g})$ & $11.4(7,17.4)$ & $8.6(4.8,11.7)$ & 0.180 & 0.297 \\
\hline Total SCFA ( $\mu \mathrm{mol} / \mathrm{g})$ & $401(386,663)$ & $395(344,537)$ & 0.533 & 0.255 \\
\hline Acetate (\%) & $60.2(56.7,65.2)$ & $71.6(60.2,75.4)$ & 0.037 & 0.027 \\
\hline Propionate (\%) & $20.6(18,21.4)$ & $18.7(12.4,20.9)$ & 0.450 & 0.208 \\
\hline Isobutyrate (\%) & $2.2(2.02,3.4)$ & $1.9(1.4,2.5)$ & 0.163 & 0.355 \\
\hline Butyrate (\%) & $15.1(11.2,17.8)$ & $8.6(8.6,9.1)$ & 0.008 & 0.016 \\
\hline Isovalerate (\%) & $2.1(1.9,3.8)$ & $1.8(1.2,3.1)$ & 0.295 & 0.598 \\
\hline
\end{tabular}

Group A: patients with faecal calprotectin levels above the median levels at food reintroduction, Group B: patients with faecal calprotectin values below the median levels at food reintroduction. Values are presented as medians (Q1, Q3). $p$ value adj: $p$ value adjusted for faecal calprotectin levels at the end of EEN. \%: proportional ratio of each SCFA to total SCFA; SCFA: Short chain fatty acids

There was no difference in faecal starch output between the two groups (median [Q1, Q3], g/100 g; Group A: $0.2[0.1,0.8]$ vs. Group B: $1.10[0.10,1.40], p=0.141$, $p$-adj $=0.573)$. 


\section{Discussion}

This study identified differences in the intake of certain nutrients, dietary patterns, and diet-related biomarkers in faeces between children with $\mathrm{CD}$ who demonstrated different levels of intestinal inflammation after food reintroduction, following successful treatment with EEN.

A higher intake of fibre and of butyrate, its proxy biomarker in faeces, was observed in patients with raised levels of FC. This observation is in contrast to the previously ascribed protective role of fibre in the development of $C D$ [3, 24, 25]. Likewise, the role of fibre in the management of IBD remains unclear. While observational research data point to a protective role of fibre in preventing a disease flare in CD [26], other data showed that a higher dietary fibre intake was positively associated with risk for clinical relapse [27]. Overall, the findings of the current study align with the lack of evidence supporting the effectiveness of fibre in the management of the disease [28-31]. A compositional analysis of 61 EEN formulas used for the induction of remission in $\mathrm{CD}$ also showed that $<20 \%$ of those formulas contained fibre [32]. Collectively, these results demonstrate that lack of fibre does not have a deleterious effect on disease activity, and indeed may have a seemingly unexpected unfavourable effect.

Patients in the current study with higher FC levels reported a higher protein and phosphorus intake, along with a higher intake of red and processed meat. Although we did not assess separately the intake of protein from animal or plant sources, the presence of a moderate-tostrong correlation between the intake of protein and meat products (spearman rho $=0.66, p=0.01$ ), but not with cereal products, nor vegetables indicates that protein from animal sources is likely to explain the negative relationship with FC we observed in the current study. High intake of animal protein has been associated with development of IBD [33,34], and in patients with ulcerative colitis (UC), high intake of protein, total, as well as red and processed meat, was positively associated with risk of relapse [15]. In a recent retrospective study, patients with IBD in clinical remission had a lower total and animal protein intake compared to patients who experienced a relapse after a 2-year follow-up [35]. In contrast, a recent RCT showed that clinical relapse rates and FC levels did not differ between patients with $C D$ who consumed at least two portions of red and processed meat per week, and others who consumed less than one serving per month [36]. The positive associations between FC and phosphorus intake may indicate an increased intake of grains and meat products which are rich in phosphorus. Although the effect of phosphorus has not been extensively explored in human IBD, dietary phosphate has been shown to be pro-inflammatory in animals [37].
Using machine learning on food group-based analysis, we showed that a dietary pattern consisting of cereal and meat-based products could successfully predict the assignment of $92 \%$ of patients into their respective FC group, with meat and cereal products showing a positive association with FC. On the contrary, the intake of eggs and fruit was associated with lower FC levels in another model with higher misclassification error though. In a recent cross-sectional study of patients with $\mathrm{CD}$, principal component analysis also identified that a dietary pattern rich in rice, pasta and red meat, among other foods, was associated with increased symptom frequency, but did not differentiate patients with active disease from those in remission [38]. In another study which assessed dietary patterns, a "Western-type" eating pattern consisting of mainly grains, red and processed meat and highsugar foods was positively associated with a higher risk for clinical relapse [35]. Of note, most novel dietary therapies for management of disease activity and intestinal inflammation in patients with CD often exclude or limit the intake of grains/gluten and red and processed meat [39]. In the current study, patients with higher FC levels also reported a higher consumption of gluten containing cereal products, which points to gluten-containing foods as potential driver of intestinal inflammation.

In addition to conventional dietary assessment, we measured diet-originating bacterial metabolites and dietary components in faeces as complementary biomarkers of consumption of certain foods previously implicated in CD pathogenesis [40]. The lack of significant differences in the levels of faecal starch between the two CD groups did not parallel the signals we observed with fibre intake. However, fibre encompasses an umbrella term of structurally diverse carbohydrates with potentially different roles in CD [41]. Although the intake of gluten containing cereals was higher in Group A, faecal GIP levels did not differ between the two groups. However, the method used to quantify faecal GIP is sensitive at detecting transgressions to gluten-free diet compliance rather than quantitatively estimating variable intakes of gluten, which might be more important in a dose-response relationship with initiation of intestinal inflammation [19].

In the current study, double levels of faecal butyrate levels were observed in patients with higher FC levels after food reintroduction. This signal was associated with higher dietary fibre intake from which butyrate originates as an end-product of bacterial fermentation in the same group (Spearman's correlation between fibre and butyrate levels: $\rho=0.55, p=0.051$ ) [42]. Higher levels of butyrate in the caecum have been shown to aggravate animal colitis [41] and a significant reduction in butyrate levels in faecal samples of children responding to EEN paralleled with a decrease in FC $[18,43]$. These data suggest that 
high amounts of butyrate are not of crucial importance for maintenance of intestinal health in patients with $\mathrm{CD}$ and its role in the disease course requires further exploration.

Although patients in Group A reported higher protein intakes compared to those in Group B, there were no significant differences in BCFA levels between the two groups. This could potentially result from adequate intake of dietary fibre in those patients, which exceeds the threshold below which excessive protein fermentation occurs in the colon [17].

The small sample size is the main limitation of this pilot study. This was due to the modest number of patients who met our stringent inclusion criteria and few patients who did not return dietary records paired with faecal samples. However, selection of a homogenous population with all patients enrolled at the end of an EEN course and while they were still in clinical remission, without receiving other concomitant induction treatment, minimised variance in our measured outcomes and increased statistical power. Beyond the dietary analysis presented here, the observed dietary signals of the current study might also represent biomarkers of unidentified covariates of other food ingredients which might be important in gut inflammation in $\mathrm{CD}$. An indicative example could be baker's yeast, which is present in bread and bakery products, constitutes a significant proportion of the cereal and cereal-based products group, and has been previously implicated in aggravating disease activity in CD [44]. It is also possible that nutrient interactions are more important than single nutrients alone in the underlying pathogenesis of CD [45]. Measurement of SCFA in faeces is the abstract of net production and absorption. However, as direct measurement of SCFA in the caecum would be almost impossible, faecal SCFA are considered suitable proxies of intestinal SCFA production; and by extension of fibre intake as has been demonstrated here by the positive associations between the amount of fibre consumed and levels of faecal SCFA [46, 47].

\section{Conclusions}

The current pilot study for first time in the literature identified that a diet higher in dietary fibre, protein, and red processed meat was associated with higher levels of intestinal inflammation after early food reintroduction in children with CD, post-EEN. These data need confirmation in larger, prospective studies to gain more insight into the dietary triggers of gut inflammation in $\mathrm{CD}$, including the role subtypes of dietary fibre, and any mediating role the microbiome may have. Such research would help identify dietary components causing inflammation in CD and enable development of food reintroduction and personalised dietary regimes, leading potentially to improvements in $\mathrm{CD}$ management and prolonged clinical remission. A clinical trial like that is currently undergoing [48].

\section{Abbreviations}

BCFA: Branched chain fatty acids; FC: Faecal calprotectin; GIP: Gluten immunogenic peptides; NDNS: National Diet and Nutrition Survey; SCFA: Short chain fatty acids.

\section{Supplementary Information}

The online version contains supplementary material available at https://doi. org/10.1186/s12876-021-02029-4.

Additional file 1: Fig. S1 (.png) Faecal calprotectin values before the start of EEN treatment and at 4 weeks and 8 weeks of EEN treatment in all 14 patients. Different colours indicate different patients.

Additional file 2: Fig. S2. (.pdf) Hierarchical dendrogram showing the classification of individual food groups to larger level food groups, based on the National Diet and Nutrition Survey

Additional file 3: Fig. S3. (.tif) Stratification of patients based on the median faecal calprotectin $(900 \mathrm{mg} / \mathrm{kg}$ ) of the entire group at food reintroduction. Group A ( $n=7)$ : above median faecal calprotectin, Group B $(n=7)$ : below median faecal calprotectin.

Additional file 4. Supplementary Tables 1, 2, 3.

\section{Acknowledgements}

Not applicable.

\section{Authors' contributions}

KG carried out the dietary analysis, parts of the statistical analysis, laboratory analysis and produced the first draft for publication. ML carried out patient recruitment, sample collection and co-ordinated most of the research activities and parts of the laboratory analysis. CMC carried out patient recruitment, sample collection and part of the laboratory analysis. BN co-ordinated and carried out part of the statistical analysis. UZI contributed to the study design, ethical application, and funding award, and statistical analysis, edited the first draft for publication and co-supervised the researcher. VS contributed to laboratory analysis and helped with data analysis and interpretation. LG performed the assessment of disease phenotype for the recruited patients and helped with patient follow-up. HD helped with the dietary aspects and analysis. VG, $L C, E B, T C, L A, C D, D M F, A B$ and RT identified and followed up patients. SM co-supervised the researcher and edited the first draft. $\mathrm{RH}$ contributed to the study design, ethical application and funding award and edited critically the first draft for publication. RKR developed the study design, funding award, ethical application, identified and followed up patients, edited critically the first draft for publication, trained the researcher in patient recruitment, cosupervised the main researcher and co-ordinated the study. K Gerasimidis conceived and developed the study design, ethical application, funding award, edited critically the first draft for publication, trained the researcher for laboratory analysis, co-supervised the main researcher and co-ordinated the study. All the authors contributed to: (1) the conception and design of the study, or acquisition of data, or analysis and interpretation of data, (2) drafting the article or revising it critically for important intellectual content, (3) final approval of the version to be submitted. All authors read and approved the final manuscript.

\section{Funding}

K. Gkikas Ph.D. studentship was funded in partnership from the University of Glasgow and Nestle Health Science. ML Ph.D. studentship was funded by the Engineering and Physical Sciences Research Council and Nestle Health Science. CMC was funded by the Ph.D. studentship from Glasgow Children's Hospital Charity. Dr. Ben Nichols was funded by a grant from the Biotechnology and Biological Sciences Research Council (BB/R006539/1) and The Catherine McEwan Foundation. The work of the IBD team in Glasgow is supported 
by the Catherine McEwan foundation. UZI is funded by NERC Independent Research Fellowship NE/L011956/1. The funding bodies had no impact on study design, analysis or conclusions reached.

\section{Availability of data and materials}

The data underlying this article will be shared on reasonable request to the corresponding author.

\section{Declarations}

\section{Ethics approval and consent to participate}

The study was approved by the NHS West of Scotland Research Ethics Committee (14/WS/1004) and participants, and their carers signed informed consent.

\section{Consent for publication}

Not applicable.

\section{Competing interests}

$\mathrm{RH}$ has received speakers/consultancy fees or conference support from Nutricia, and 4D Pharma. RKR has received speaker's fees, travel support and/ or participated in medical board meetings with Nestle, MSD Immunology, AbbVie, Dr Falk, Takeda, Napp, Mead Johnson, Nutricia \& 4D Pharma. RH \& RKR are supported by an NHS Research Scotland Senior Career Researcher Fellowship. K Gerasimidis has received research grants, speakers' fees and had conference attendance paid by Nutricia/Nestle, Nutricia-Danone, Baxter, Abbott, and Dr Falk. The rest of the authors (K Gkikas, ML, BN, UZI, CMC, VS, LG, HD, VG, LC, $E B, T C, L A, C D, D M F, A R B, R T$ and $S M$ ) have no competing interests to report.

\section{Guidelines statement}

All methods were carried out in accordance with relevant guidelines and regulations.

\section{Author details}

${ }^{1}$ Human Nutrition, School of Medicine, Dentistry and Nursing, College of Medical, Veterinary and Life Sciences, University of Glasgow, Glasgow, UK. ${ }^{2}$ Civil Engineering, School of Engineering, University of Glasgow, Glasgow, UK. ${ }^{3}$ Department of Paediatric Gastroenterology, Hepatology and Nutrition, Royal Hospital for Children, Glasgow, UK. ${ }^{4}$ Department of Paediatrics, Crosshouse Hospital, Kilmarnock, UK. ${ }^{5}$ Department of Paediatrics, Wishaw General Hospital, Wishaw, UK. ${ }^{6}$ Institute for Infection, Immunity and Inflammation, University of Glasgow, Glasgow, UK. ${ }^{7}$ Royal Hospital for Children and Young People, Edinburgh, UK.

Received: 22 June 2021 Accepted: 2 November 2021

Published online: 03 December 2021

\section{References}

1. Ng SC, Shi HY, Hamidi N, Underwood FE, Tang W, Benchimol El, et al. Worldwide incidence and prevalence of inflammatory bowel disease in the 21 st century: a systematic review of population-based studies. Lancet. 2018;390(10114):2769-78.

2. Khalili H, Håkansson N, Chan SS, Chen Y, Lochhead P, Ludvigsson JF, et al. Adherence to a Mediterranean diet is associated with a lower risk of later-onset Crohn's disease: results from two large prospective cohort studies. Gut. 2020;69(9):1637-44.

3. Ananthakrishnan AN, Khalili H, Konijeti GG, Higuchi LM, de Silva P, Korzenik JR, et al. A prospective study of long-term intake of dietary fiber and risk of Crohn's disease and ulcerative colitis. Gastroenterology. 2013;145(5):970-7.

4. Levine A, Sigall Boneh R, Wine E. Evolving role of diet in the pathogenesis and treatment of inflammatory bowel diseases. Gut. 2018;67(9):1726-38.

5. Ruemmele FM, Veres G, Kolho KL, Griffiths A, Levine A, Escher JC, et al. Consensus guidelines of ECCO/ESPGHAN on the medical management of pediatric Crohn's disease. J Crohns Colitis. 2014;8(10):1179-207.
6. Narula N, Dhillon A, Zhang D, Sherlock ME, Tondeur M, Zachos M. Enteral nutritional therapy for induction of remission in Crohn's disease. Cochrane Database Syst Rev. 2018;4(4):Cd000542.

7. Logan M, ljaz UZ, Hansen R, Gerasimidis K, Russell RK. Letter: reproducible evidence shows that exclusive enteral nutrition significantly reduces faecal calprotectin concentrations in children with active Crohn's disease. Aliment Pharmacol Ther. 2017;46(11-12):1119-20.

8. Gerasimidis K, Nikolaou CK, Edwards CA, McGrogan P. Serial fecal calprotectin changes in children with Crohn's disease on treatment with exclusive enteral nutrition: associations with disease activity, treatment response, and prediction of a clinical relapse. J Clin Gastroenterol. 2011;45(3):234-9.

9. Logan M, Clark CM, ljaz UZ, Gervais L, Duncan H, GarrickV, et al. The reduction of faecal calprotectin during exclusive enteral nutrition is lost rapidly after food re-introduction. Aliment Pharmacol Ther. 2019;50(6):664-74.

10. Turner D, Levine A, Walters TD, Focht G, Otley A, López VN, et al. Which PCDAI Version Best Reflects Intestinal Inflammation in Pediatric Crohn Disease? J Pediatr Gastroenterol Nutr. 2017;64(2):254-60.

11. Levine A, Griffiths A, Markowitz J, Wilson DC, Turner D, Russell RK, et al. Pediatric modification of the Montreal classification for inflammatory bowel disease: the Paris classification. Inflamm Bowel Dis. 2011;17(6):1314-21.

12. England PH. NDNS: time trend and income analyses for Years 1 to 9. 2019.

13. Research WCRFAIfC. Diet, nutrition, physical activity and cancer: a global perspective. Continuous Update Project Expert Report 2018.; 2018.

14. Maconi G, Ardizzone S, Cucino C, Bezzio C, Russo AG, Bianchi PG. Pre-illness changes in dietary habits and diet as a risk factor for inflammatory bowel disease: a case-control study. World J Gastroenterol. 2010;16(34):4297-304.

15. Jowett SL, Seal CJ, Pearce MS, Phillips E, Gregory W, Barton JR, et al. Influence of dietary factors on the clinical course of ulcerative colitis: a prospective cohort study. Gut. 2004;53(10):1479-84.

16. Dietary reference values for food energy and nutrients for the United Kingdom. In: Report of the Panel on Dietary Reference Values of the Committee on Medical Aspects of Food Policy. Rep Health Soc Subj (Lond). 1991;41:1-210.

17. Svolos V, Hansen R, Nichols B, Quince C, ljaz UZ, Papadopoulou RT, et al. Treatment of active Crohn's disease with an ordinary food-based diet that replicates exclusive enteral nutrition. Gastroenterology. 2019;156(5):135467.e6.

18. Gerasimidis K, Bertz M, Hanske L, Junick J, Biskou O, Aguilera M, et al. Decline in presumptively protective gut bacterial species and metabolites are paradoxically associated with disease improvement in pediatric Crohn's disease during enteral nutrition. Inflamm Bowel Dis. 2014;20(5):861-71.

19. Gerasimidis K, Zafeiropoulou K, Mackinder M, ljaz UZ, Duncan H, Buchanan E, et al. Comparison of clinical methods with the faecal gluten immunogenic peptide to assess gluten intake in coeliac disease. J Pediatr Gastroenterol Nutr. 2018;67(3):356-60.

20. Kassambara A. Machine Learning Essentials: Practical Guide in R 2018.

21. Thomas Lumley based on Fortran code by Alan Miller. leaps: Regression Subset Selection. R package version 3 2017. https://CRAN.R-project.org/ package=leaps.

22. Kuhn M. Building predictive models in R using the caret package. J Stat Softw. 2008;28(5):1-26.

23. Liaw A, Wiener M. Classification and regression by random Forest. R News. 2002;2(3):18-22.

24. Hou JK, Abraham B, El-Serag H. Dietary intake and risk of developing inflammatory bowel disease: a systematic review of the literature. Am J Gastroenterol. 2011;106(4):563-73.

25. Liu X, Wu Y, Li F, Zhang D. Dietary fiber intake reduces risk of inflammatory bowel disease: result from a meta-analysis. Nutr Res. 2015;35(9):753-8.

26. Brotherton CS, Martin CA, Long MD, Kappelman MD, Sandler RS. Avoidance of fiber is associated with greater risk of Crohn's disease flare in a 6-month period. Clin Gastroenterol Hepatol. 2016;14(8):1130-6.

27. Opstelten $J L$, de Vries JHM, Wools A, Siersema PD, Oldenburg B, Witteman BJM. Dietary intake of patients with inflammatory bowel disease: a comparison with individuals from a general population and associations with relapse. Clin Nutr. 2019;38(4):1892-8. 
28. Wedlake L, Slack N, Andreyev HJ, Whelan K. Fiber in the treatment and maintenance of inflammatory bowel disease: a systematic review of randomized controlled trials. Inflamm Bowel Dis. 2014;20(3):576-86.

29. Limketkai BN, Iheozor-Ejiofor Z, Gjuladin-Hellon T, Parian A, Matarese LE, Bracewell K, et al. Dietary interventions for induction and maintenance of remission in inflammatory bowel disease. Cochrane Database Syst Rev. 2019;2(2):Cd012839.

30. Jones VA, Dickinson RJ, Workman E, Wilson AJ, Freeman AH, Hunter JO. Crohn's disease: maintenance of remission by diet. Lancet. 1985;2(8448):177-80.

31. Gkikas K, Gerasimidis K, Milling S, ljaz UZ, Hansen R, Russell RK. Dietary strategies for maintenance of clinical remission in inflammatory bowel diseases: are we there yet? Nutrients. 2020;12(7):201.

32. Logan M, Gkikas K, Svolos V, Nichols B, Milling S, Gaya DR, et al. Analysis of 61 exclusive enteral nutrition formulas used in the management of active Crohn's disease - new insights into dietary disease triggers. Aliment Pharmacol Ther. 2020;51(10):935-47.

33. Jantchou P, Morois S, Clavel-Chapelon F, Boutron-Ruault MC, Carbonnel F. Animal protein intake and risk of inflammatory bowel disease: the E3N prospective study. Am J Gastroenterol. 2010;105(10):2195-201.

34. Shoda R, Matsueda K, Yamato S, Umeda N. Therapeutic efficacy of N-3 polyunsaturated fatty acid in experimental Crohn's disease. J Gastroenterol. 1995;30(Suppl 8):98-101.

35. Peters V, Spooren C, Pierik MJ, Weersma RK, van Dullemen HM, Festen EAM, et al. Dietary intake pattern is associated with occurrence of flares in IBD patients. J Crohns Colitis. 2021;15(8):1305-15.

36. Albenberg L, Brensinger CM, Wu Q, Gilroy E, Kappelman MD, Sandler $\mathrm{RS}$, et al. A diet low in red and processed meat does not reduce rate of Crohn's disease flares. Gastroenterology. 2019;157(1):128-36.e5.

37. Sugihara K, Masuda M, Nakao M, Abuduli M, Imi Y, Oda N, et al. Dietary phosphate exacerbates intestinal inflammation in experimental colitis. $J$ Clin Biochem Nutr. 2017;61(2):91-9.

38. de Castro MM, Corona LP, Pascoal LB, Miyamoto JÉ, Ignacio-Souza LM, de Lourdes Setsuko Ayrizono M, et al. Dietary patterns associated to clinical aspects in Crohn's disease patients. Sci Rep. 2020;10(1):7033.

39. Sabino J, Lewis JD, Colombel JF. Treating inflammatory bowel disease with diet: a taste test. Gastroenterology. 2019;157(2):295-7.

40. Lee D, Albenberg L, Compher C, Baldassano R, Piccoli D, Lewis JD, et al. Diet in the pathogenesis and treatment of inflammatory bowel diseases. Gastroenterology. 2015;148(6):1087-106.

41. Singh V, Yeoh BS, Walker RE, Xiao X, Saha P, Golonka RM, et al. Microbiota fermentation-NLRP3 axis shapes the impact of dietary fibres on intestinal inflammation. Gut. 2019;68(10):1801-12.

42. Albenberg LG, Wu GD. Diet and the intestinal microbiome: associations, functions, and implications for health and disease. Gastroenterology. 2014;146(6):1564-72.

43. Walton C, Montoya MP, Fowler DP, Turner C, Jia W, Whitehead RN, et al. Enteral feeding reduces metabolic activity of the intestinal microbiome in Crohn's disease: an observational study. Eur J Clin Nutr. 2016;70(9):1052-6.

44. Barclay GR, McKenzie H, Pennington J, Parratt D, Pennington CR. The effect of dietary yeast on the activity of stable chronic Crohn's disease Scand J Gastroenterol. 1992;27(3):196-200.

45. Llewellyn SR, Britton GJ, Contijoch EJ, Vennaro OH, Mortha A, Colombel $J F$, et al. Interactions between diet and the intestinal microbiota alter intestinal permeability and colitis severity in mice. Gastroenterology. 2018;154(4):1037-46.e2.

46. McOrist AL, Miller RB, Bird AR, Keogh JB, Noakes M, Topping DL, et al. Fecal butyrate levels vary widely among individuals but are usually increased by a diet high in resistant starch. J Nutr. 2011;141(5):883-9.

47. Whelan K, Judd PA, Preedy VR, Simmering R, Jann A, Taylor MA. Fructooligosaccharides and fiber partially prevent the alterations in fecal microbiota and short-chain fatty acid concentrations caused by standard enteral formula in healthy humans. J Nutr. 2005;135(8):1896-902.

48. ClinicalTrials.gov U.S. National Library of Medicine. NCT00287391: The Intensive Post Exclusive Enteral Nutrition Study (iPENS) 2020. https://clini caltrials.gov/ct2/show/NCT04225689.

\section{Publisher's Note}

Springer Nature remains neutral with regard to jurisdictional claims in published maps and institutional affiliations.

Ready to submit your research? Choose BMC and benefit from:

- fast, convenient online submission

- thorough peer review by experienced researchers in your field

- rapid publication on acceptance

- support for research data, including large and complex data types

- gold Open Access which fosters wider collaboration and increased citations

- maximum visibility for your research: over $100 \mathrm{M}$ website views per year

At BMC, research is always in progress.

Learn more biomedcentral.com/submissions 